\title{
Routine Duodenal Biopsies in the Absence of Endoscopic Markers of Celiac Disease Are Not Useful: An Observational Study
}

\author{
Fernando Castro, ${ }^{1}$ Jennifer Shiroky, ${ }^{1}$ Ramu Raju, ${ }^{2}$ Einar Lurix, ${ }^{3}$ Tolga Erim, \\ Yasmin Johnston, ${ }^{4}$ and Andrew Ukleja ${ }^{1}$ \\ ${ }^{1}$ Department of Gastroenterology, Cleveland Clinic Florida, Weston, FL 33331, USA \\ ${ }^{2}$ Gastroenterology of the Rockies, Boulder, CO 80303, USA \\ ${ }^{3}$ Gastroenterology Consultants, IMA Greenville Hospital System, Greenville, SC 29607, USA \\ ${ }^{4}$ Department of Pathology, Cleveland Clinic Florida, Weston, FL 33331, USA
}

Correspondence should be addressed to Fernando Castro; castrof@ccf.org

Received 5 June 2013; Accepted 2 July 2013

Academic Editors: P. Born, B. Braden, G. Larciprete, and M. Sewitch

Copyright (C) 2013 Fernando Castro et al. This is an open access article distributed under the Creative Commons Attribution License, which permits unrestricted use, distribution, and reproduction in any medium, provided the original work is properly cited.

Background. European studies have shown the utility of limiting endoscopic biopsies to diagnose celiac disease (CD) to patients that have high-risk symptoms or present with positive serology. However, many centers in the U.S. have open access endoscopy. Patients are referred without prior serologic testing, and endoscopists often decide whether or not to biopsy at the time of procedure. Aims. Evaluate the yield of duodenal biopsies for the diagnosis of CD in patients undergoing upper endoscopy without prior serologic testing. Methods. This retrospective study evaluated the frequency of CD diagnosis based duodenal biopsies. Researchers were interested in the yield of endoscopic stigmata findings in patients with high-risk symptoms versus low risk. Results. Eight hundred and ten patients met entry criteria at the Cleveland Clinic Florida between 2004 and 2008; 320 presented with highrisk symptoms; and 490 low risk. Sixty-one (7.5\%) displayed endoscopic stigmata, and 10/61 (16.3\%) were diagnosed with CD. Only patients who exhibited endoscopic stigmata were later diagnosed based on histologic findings. The presence of endoscopic stigmata greatly increased the probability of diagnosing CD, with a positive likelihood ratio of 15.6. Conclusions. When performing upper endoscopy without known serological markers for CD, clinicians should limit duodenal biopsies to patients with high-risk symptoms or endoscopic stigmata.

\section{Introduction}

The diverse presentations of celiac disease (CD) often delay diagnosis. Although serological tests are reliable, endoscopic biopsies are essential in diagnosing the disease because they allow for direct observation of histologic abnormalities compatible with CD [1-6]. Therefore some centers encourage routine endoscopic biopsies in all patients with upper gastrointestinal symptomatology $[7,8]$. One advantage of early diagnosis and treatment can be reduced medical costs. However, excessive biopsying of endoscopic patients can lead to unnecessary medical expenditures. Therefore developing stricter guidelines for biopsying to rule out CD may help reduce endoscopy costs and waste.

Hopper et al. found that restricting duodenal biopsies to only patients that presented with high-risk symptoms of $\mathrm{CD}$ as defined by weight loss, anemia, diarrhea, or a positive immunoglobulin tissue transglutaminase antibody (IgA tTGA) serology would have successfully diagnosed all individuals who had the disease [8]. Our study examines the effectiveness of duodenal biopsying in patients with whom the presence of IgA tTGA, or other serologic markers, is unknown, a scenario more applicable to medical practices in the United States. 
Celiac disease can cause mucosal inflammation and lesions that can be identified endoscopically. For example, scalloping or loss of duodenal folds, mosaic pattern, and nodularity can serve as endoscopic markers of the disease $[6,7,9,10]$. These observations may indicate a need for duodenal biopsies in patients that present both typical and atypical presentations of CD. However, assessments of the sensitivity and specificity of endoscopic stigmata have varied greatly. Most studies were conducted in Europe around 10 years ago, and there is currently limited data on the usefulness of endoscopic stigmata in the United States' population. This study aimed to determine the utility of duodenal endoscopic markers in diagnosing CD in patients in the United States with no prior serologic workup.

\section{Methods}

2.1. Participants. Our study received approval from the IRB in October 2005. Researchers reviewed upper endoscopy records from January 2004 to December 2008 at the Cleveland Clinic Florida in order to identify patients who had received distal or descending duodenal biopsies. Clinical history, indication for endoscopy, endoscopic appearance of the duodenum, and histologic findings were reviewed. Patients who had an established CD diagnosis, family history of the condition, had any celiac serologic markers performed prior to the endoscopy, or had biopsies of a duodenal polyp, mass, or ulceration were excluded from the study.

2.2. Materials. Endoscopic examinations were performed with a standard definition videoendoscope (Pentax Corporation, Tokyo, Japan) until October 2007. A high definition endoscope (FUJINON Corporation, Minatoku, Tokyo, Japan) was used in subsequent examinations. Mucosal samples were obtained by using a disposable standard size biopsy forceps (Boston Scientific, Doral, Florida).

2.3. Methods. Clinical presentations were classified as either high or low risk. High-risk factors included: weight loss, diarrhea, or anemia. Low-risk factors included all other patients, some of which exhibited gastroesophageal reflux disease (GERD), dysmotility-like dyspepsia, and ulcer-like dyspepsia.

The endoscopic appearance of $\mathrm{CD}$ was classified as scalloping, blunting of folds, nodularity, fissuring, or mosaic patterns in the duodenal mucosa. The gastroenterology department's routine practice involved obtaining at least four duodenal biopsies as per guideline [10]. Five experienced gastroenterologists, who had performed at least 1,000 endoscopies prior, performed all the procedures.

Biopsies were fixed in formalin, routinely processed, and then stained with hematoxylin, eosin stain, and Schiff periodic acid. Pathologists were presented with a limited clinical history and asked to evaluate the specimens for CD. It was a common practice that pathologists were blind to endoscopic appearance. The pathologic diagnosis was made based on presence of intraepithelial lymphocytosis and partial or total villous atrophy. Biopsies with abnormal histology were then
TABLE 1: Demographics of patients.

\begin{tabular}{lcc}
\hline & Demographics & $\%$ \\
\hline Gender & $n$ & \\
Male & 287 & 35.3 \\
Female & 523 & 64.6 \\
Age (SD) & & \\
Mean & $49( \pm 15.7)$ & \\
Male & $52( \pm 16.3)$ & \\
Female & $48( \pm 15.3)$ & \\
Race & & 4.4 \\
African American & 36 & 1.6 \\
Asian & 13 & 62.2 \\
Caucasian & 504 & 24.8 \\
Hispanic & 201 & 0.9 \\
Native American & 7 & 5.7 \\
Other & 46 & 0.4 \\
Unknown & 3 & \\
\hline
\end{tabular}

TABLE 2: Endoscopic stigmata findings.

\begin{tabular}{lccc}
\hline & \multicolumn{2}{c}{ Endoscopic findings } & \multirow{2}{*}{ Celiac patients } \\
& Number total & $\%$ & \\
\hline Scalloping & 28 & 45.9 & 5 \\
Blunting/flattened & 22 & 36.1 & 1 \\
Fissuring & 1 & 1.6 & 0 \\
Mosaic & 1 & 1.6 & 0 \\
Nodular & 9 & 14.8 & 4 \\
\hline Total & 61 & & 10 \\
\hline
\end{tabular}

reviewed by another pathologist for accuracy and classified according to the Marsh classification.

\section{Results}

We identified 911 patients from whom duodenal biopsies had been obtained. Of these, 810 patients met the study's criteria, 523 females and 287 males. Sixty-two percent of the subjects were Caucasians, 25\% Hispanic, and 13\% identified with other ethnicities (Table 1).

A total of $320(39.5 \%)$ presented with high-risk symptoms, while 490 (60.5\%) displayed low-risk symptoms. The most common symptoms were GERD (30.6\%), anemia (18.5\%), and diarrhea (16.2\%). Sixty-one patients (7.5\%) had abnormal endoscopic findings suggestive of CD. These optical observations included scalloping (45.9\%), blunting or flattening folds $(36.1 \%)$, nodular mucosa (14.8\%), fissuring (1.6\%), and a mosaic pattern (1.6\%) (Table 2).

Among patients with low-risk symptoms, 37 (7.6\%) had endoscopic findings suggestive of CD. Endoscopists observed atypical duodenal mucosa in 14/248 (5.6\%) patients with GERD, 17/55 (30.9\%) with ulcer-like dyspepsia, 3/21 (14.5\%) with dysmotility-like dyspepsia, and 1/16 (6.3\%) with other atypical symptoms. Three patients ( $8 \%$ ) with low-risk symptoms and endoscopic findings were positive for CD based 
TABle 3: Patients diagnosed with celiac disease.

\begin{tabular}{|c|c|c|c|c|c|c|c|}
\hline \multirow{2}{*}{ Age } & \multirow{2}{*}{ Gender } & \multicolumn{4}{|c|}{ Patients with celiac disease } & \multirow{2}{*}{$\begin{array}{l}\text { Improvement } \\
\text { with diet }\end{array}$} & \multirow{2}{*}{$\begin{array}{l}\text { Marsh } \\
\text { Score }\end{array}$} \\
\hline & & Ethnicity & $\begin{array}{l}\text { Indication for } \\
\text { endoscopy }\end{array}$ & Endoscopic appearance & Positive markers & & \\
\hline 47 & $\mathrm{~F}$ & Hispanic/Latino & Anemia & Nodular & Ttg-IgA & Yes & $3 b$ \\
\hline 77 & M & Caucasian & Anemia & Flattening & Ttg-IgA & $\begin{array}{l}\text { No } \mathrm{f} / \mathrm{u} \text { to } \\
\text { determine }\end{array}$ & $3 a$ \\
\hline 37 & $\mathrm{~F}$ & Hispanic/Latino & Anemia & Flattening and scalloping & $\begin{array}{l}\text { Ttg-IgA, } \\
\text { endomysial IgA }\end{array}$ & Yes & $3 a$ \\
\hline 42 & M & Caucasian & Diarrhea, weight loss & $\begin{array}{l}\text { Nodular, granular, } \\
\text { flattening with white } \\
\text { patchy exudate }\end{array}$ & $\begin{array}{c}\text { Ttg-IgA, } \\
\text { endomysial IgA }\end{array}$ & Yes & $3 c$ \\
\hline 35 & $\mathrm{~F}$ & Caucasian & Diarrhea, weight loss & Scalloping & Gliadin IgG & Yes & $3 a$ \\
\hline 57 & M & Hispanic/Latino & Epigastric pain & $\begin{array}{c}\text { Nodular, inflammation } \\
\text { and erosion }\end{array}$ & Ttg-IgA & $\begin{array}{l}\text { No } \mathrm{f} / \mathrm{u} \text { to } \\
\text { determine }\end{array}$ & $3 a$ \\
\hline 50 & M & Hispanic/Latino & Epigastric pain & $\begin{array}{l}\text { Inflammation, erythema, } \\
\text { scalloping }\end{array}$ & $\begin{array}{l}\text { Ttg-IgA, } \\
\text { endomysial IgA }\end{array}$ & $\begin{array}{l}\text { Did not } \\
\text { follow diet }\end{array}$ & $3 b$ \\
\hline 53 & $\mathrm{~F}$ & Hispanic/Latino & Bloating & Nodular, mosaic pattern & Gliadin IgG & Yes & $3 b$ \\
\hline 44 & M & Caucasian & Diarrhea & Scalloping & $\begin{array}{l}\text { Ttg-IgA, Gliadin } \\
\text { IgA }\end{array}$ & Yes & $3 b$ \\
\hline 64 & M & Caucasian & Anemia & Scalloping & $\begin{array}{c}\text { Ttg-IgA, } \\
\text { endomysial IgA, } \\
\text { Gliadin IgA and } \\
\text { IgG }\end{array}$ & Yes & $3 c$ \\
\hline
\end{tabular}

on histological appearance and positive markers. No patients with low-risk symptoms and normal endoscopic findings were diagnosed with $\mathrm{CD}$.

Among patients with high-risk symptoms, 24 (7.5\%) had endoscopic findings suggestive of CD. Abnormal duodenal mucosa was observed in 6/131 (4.6\%) patients with diarrhea, $2 / 39(5.1 \%)$ with weight loss, and $16 / 150(10.7 \%)$ with anemia. Seven of the 24 (29\%) patients with high-risk symptoms and endoscopic findings were diagnosed with CD based on histological appearance and positive markers (Table 3).

A positive diagnosis of CD was made in 10 out of the 810 individuals. The overall yield was $1.23 \%$. Seven $(7 / 320$, $2.2 \%)$ of these patients presented with high-risk symptoms, 3 with diarrhea, and 4 with anemia. Three $(3 / 490,0.6 \%)$ presented with low risk, 2 with ulcer-like dyspepsia, and 1 with dysmotility-like dyspepsia.

There was no significant difference in the rate of detection of CD between standard definition and high definition endoscopes. The overall sensitivity of endoscopic stigmata of CD was $100 \%$ with a specificity of $93.6 \%$. The positive predictive value was $16.3 \%$, and the negative predictive value was $100 \%$. The presence of endoscopic stigmata greatly increased the probability of diagnosing $\mathrm{CD}$, with a positive likelihood ratio of 15.6 .

\section{Discussion}

This study aimed to investigate endoscopic practice patterns in patients that are suspected of having CD and have no previous serologic workup. All patients who were diagnosed with CD had stigmata upon endoscopic observation. Based on its findings, limiting routine biopsies to patients with classic or subtle endoscopic stigmata may be a reliable method for identifying CD in patients with low- and highrisk gastrointestinal symptoms.

A recent study by Hopper et al. in a British population found that performing endoscopic biopsies only in patients with classical symptoms or those with atypical symptoms and a positive TTG would have detected all cases of CD [8]. However, we are often faced with a different scenario in which patients referred for open access endoscopy do not have known prior serological markers. A decision has to be made at the time of endoscopy whether to perform biopsies on these patients.

In our study, $2.2 \%$ of patients identified as high risk were diagnosed with CD identifying this subgroup of patients as being more likely to have CD. This prevalence rate is similar to $3.0 \%$ in an at-risk population reported by Hin et al. in a pilot case-finding study in the United Kingdom [11]. Among patients with anemia, $2.7 \%$ were diagnosed with CD identifying this subgroup of patients at higher risk. However, when it comes to patients with a low-risk classification, we found the yield of routine duodenal biopsies to be only $0.6 \%$. In a large Finnish study, the yield of CD when duodenal biopsies were performed routinely during open access endoscopy was $0.6 \%$ of 3000 patients with GERD symptoms and $0.8 \%$ of 5000 patients with dyspepsia [12]. A recent British study found a similar prevalence of $0.5 \%$ in over 1200 patients with atypical symptoms [8]. Our low-risk patient yield is very similar to $0.71 \%$ prevalence in the United States estimated by a recent study by Rubio-Tapia et al. [6]. Although we found a similar prevalence of endoscopic stigmata in patients with high and low risk symptoms, endoscopic markers were less likely to signify CD in the low-risk group. Therefore, 
whenever possible serological testing should be done prior to endoscopy and biopsies targeted only to those patients with positive serology or classic endoscopic stigmata.

In this study, all patients diagnosed with CD had endoscopic markers suggestive of the disease. The endoscopic stigmata indicated a sensitivity of $100 \%$ and a specificity of $93.6 \%$. Early studies evaluating sensitivity of endoscopic stigmata found similar sensitivity of $82 \%$ for any endoscopic marker. However, subsequent studies reported lower endoscopic marker sensitivity, even as low as 50\%. Much of this research looked at the utility of endoscopic markers in patients with dyspepsia $[2,3,5,7]$. Bardella et al. found endoscopic markers to be only $50 \%$ positive in a prospective study of 517 patients. It is interesting to note that only $1 \%$ of their patients were found to have endoscopic stigmata of $\mathrm{CD}$, whereas we describe stigmata in $7.5 \%$.

The higher sensitivity of our study may be attributable to its retrospective design. The endoscopists were blind to the study potential of their procedures and were therefore not searching for strictly defined endoscopic stigmata at the time of the procedure. They may have been less rigid than those in prospective studies. A prospective study design defines stigmata beforehand, often using classic examples that may exclude other abnormal mucosa presentations. Being more selective limits the number of patients that are biopsied and may consequently diminish sensitivity.

Although retrospective studies are often seen as a limitation, there is an advantage of having used a retrospective design. Endoscopists were unaware of the study and therefore did not consciously search for strictly defined endoscopic stigmata. Thus, this design is more consistent with a real-life experience.

A common potential limitation of CD studies such as this is underestimating the actual incidence of the disease. It is recognized that patients with $\mathrm{CD}$ and mild symptoms may exhibit mild histological changes such as intraepithelial lymphocytic infiltration without villous atrophy. Only patients whose histological workup displayed criteria of intraepithelial lymphocytes with villous atrophy were classified as having CD. Therefore mild cases of the disease may have been missed. Unfortunately loosening this criteria risks diagnosing patients with CD who did not have the condition.

Given our low incidence of CD in patients with atypical symptoms and the sensitivity of endoscopic stigmata, performing biopsies in all patients with atypical symptoms does not seem to be cost effective. Our findings suggest that we should biopsy only those with atypical symptoms that exhibit endoscopic stigmata. This would represent substantial cost savings including the cost of the biopsy forceps and the cost of the pathology specimen interpretation as these amount to approximately $\$ 200$ per Medicare reimbursement rate for 2011.

Recent research has shown that early diagnosis and treatment of $\mathrm{CD}$ are associated with reduced medical costs for celiac patients up to $\$ 1764$ in the year following diagnosis [13]. As increasing efforts are made to decrease medical costs, more attention will be focused on the cost effectiveness of routine endoscopic biopsies. Contrary to prospective studies, we were able to identify all our patients with low- and high-risk classifications for CD using endoscopic stigmata. Our findings support the recommendations by the American Gastroenterology Association and the British Society of Gastroenterology to obtain biopsies in all patients with diarrhea, anemia, and weight loss given the higher incidence of CD. We also suggest that biopsies should be obtained in patients with low-risk symptoms who present with endoscopic stigmata of CD even though at times these changes may be very subtle. Prospective multicenter studies or large database results are needed to strengthen our findings.

\section{Conflict of Interests}

The authors declare that there is no conflict of interests.

\section{Authors' Contributions}

Drs. Fernando Castro, Ramu Raju, Einar Lurix, Tolga Erim, and Andrew Ukleja were the treating physicians. Dr. Johnston reviewed the biopsies. Drs. Lurix and Raju and Jennifer Shiroky wrote the paper. Drs. Fernando Castro and Tolga Erim and Jennifer Shiroky helped to analyze the data and edit the paper. All of the authors have approved the final draft submitted.

\section{References}

[1] W. Dickey and D. Hughes, "Erosions in the second part of the duodenum in patients with villous atrophy," Gastrointestinal Endoscopy, vol. 59, no. 1, pp. 116-118, 2004.

[2] A. C. Ford, E. Ching, and P. Moayyedi, "Meta-analysis: yield of diagnostic tests for coeliac disease in dyspepsia," Alimentary Pharmacology and Therapeutics, vol. 30, no. 1, pp. 28-36, 2009.

[3] S. Lecleire, F. Di Fiore, M. Antoniette et al., "Endoscopic markers of villous atrophy are not useful for the detection of celiac disease in patients with dyspeptic symptoms," Endoscopy, vol. 38, no. 7, pp. 696-701, 2006.

[4] A. K. Mandal, I. Mehdi, S. K. Munshi, and T. C. N. Lo, "Value of routine duodenal biopsy in diagnosing coeliac disease in patients with iron deficiency anaemia," Postgraduate Medical Journal, vol. 80, no. 946, pp. 475-477, 2004.

[5] G. Olds, R. McLoughlin, C. O’Morian, and M. V. Sivak, "Celiac disease for the endoscopist," Gastrointestinal Endoscopy, vol. 56, pp. 407-415, 2001.

[6] A. Rubio-Tapia, J. F. Ludbigsson, and T. L. Brantner, "The prevalence of celiac disease in the United States," American Journal of Gastroenterology, vol. 107, pp. 1538-1544, 2012.

[7] P. H. R. Green and J. A. Murray, "Routine duodenal biopsies to exclude celiac disease?” Gastrointestinal Endoscopy, vol. 58, no. 1, pp. 92-95, 2003.

[8] A. D. Hopper, S. S. Cross, D. P. Hurlstone et al., "Pre-endoscopy serological testing for coeliac disease: evaluation of a clinical decision tool," British Medical Journal, vol. 334, article 729, 2007.

[9] W. Dickey and D. Hughes, "Disappointing sensitivity of endoscopic markers for villous atrophy in a high-risk population: implications for celiac disease diagnosis during routine endoscopy," American Journal of Gastroenterology, vol. 96, no. 7, pp. 2126-2128, 2001.

[10] B. Lebwohl, R. C. Kapel, A. I. Neugut, P. H. R. Green, and R. M. Genta, "Adherence to biopsy guidelines increases celiac disease 
diagnosis," Gastrointestinal Endoscopy, vol. 74, no. 1, pp. 103-109, 2011.

[11] H. Hin, G. Bird, P. Fisher, N. Mahy, and D. Jewell, "Coeliac disease in primary care: case finding study," British Medical Journal, vol. 318, no. 7177, pp. 164-167, 1999.

[12] P. Collin, M. Rasmussen, S. Kyrönpalo, P. Laippala, and K. Kaukinen, "The hunt for coeliac disease in primary care," QJM, vol. 95, no. 2, pp. 75-77, 2002.

[13] K. H. Long, A. Rubio-Tapia, A. E. Wagie et al., "The economics of coeliac disease: a population-based study," Alimentary Pharmacology and Therapeutics, vol. 32, no. 2, pp. 261-269, 2010. 


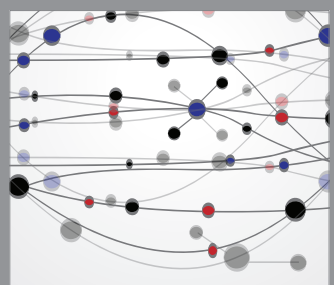

The Scientific World Journal
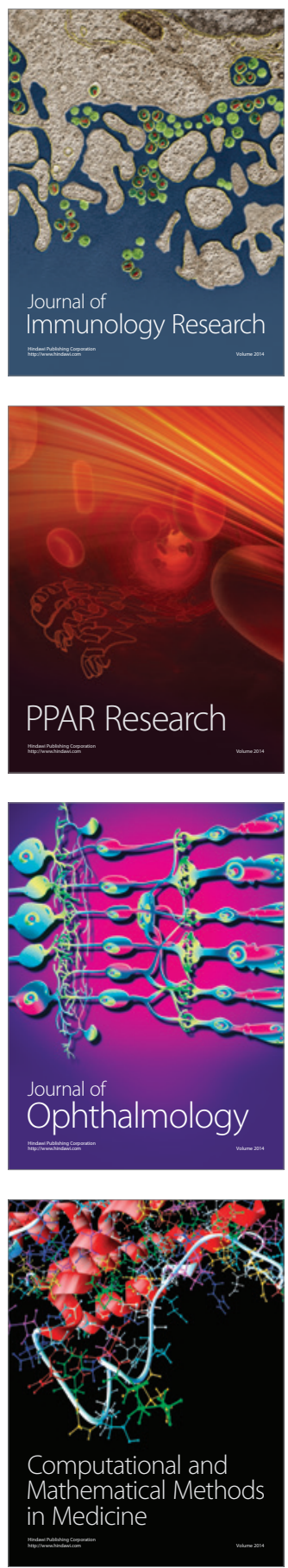

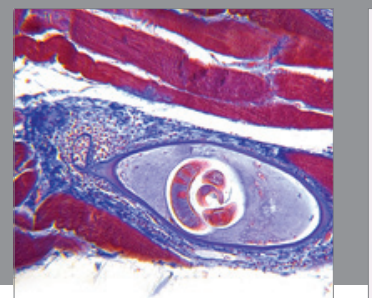

Gastroenterology

Research and Practice
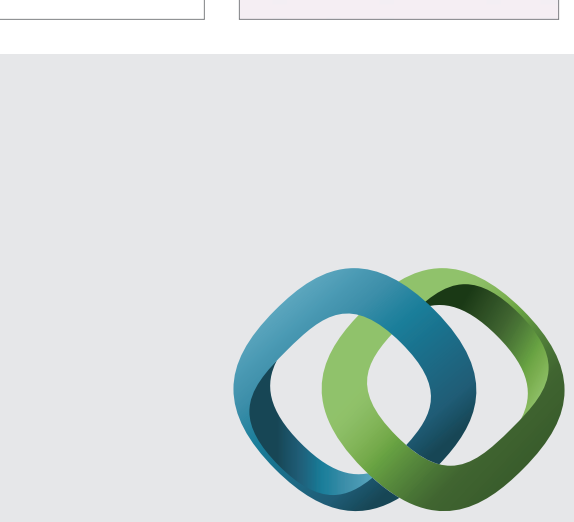

\section{Hindawi}

Submit your manuscripts at

http://www.hindawi.com
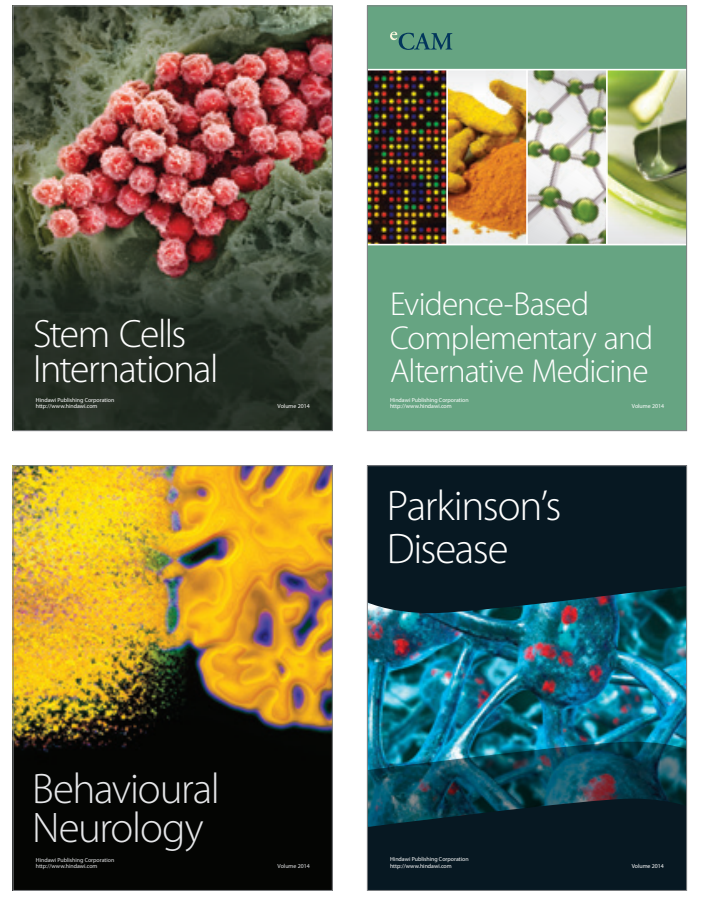
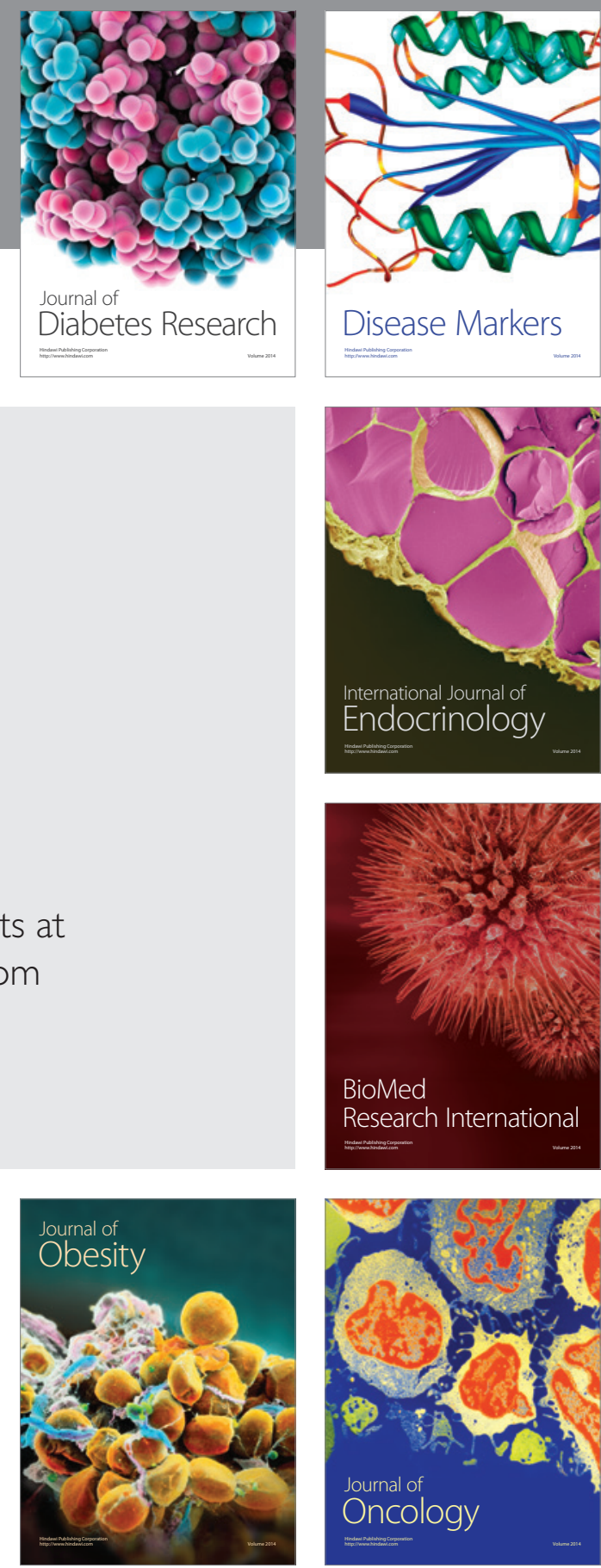

Disease Markers
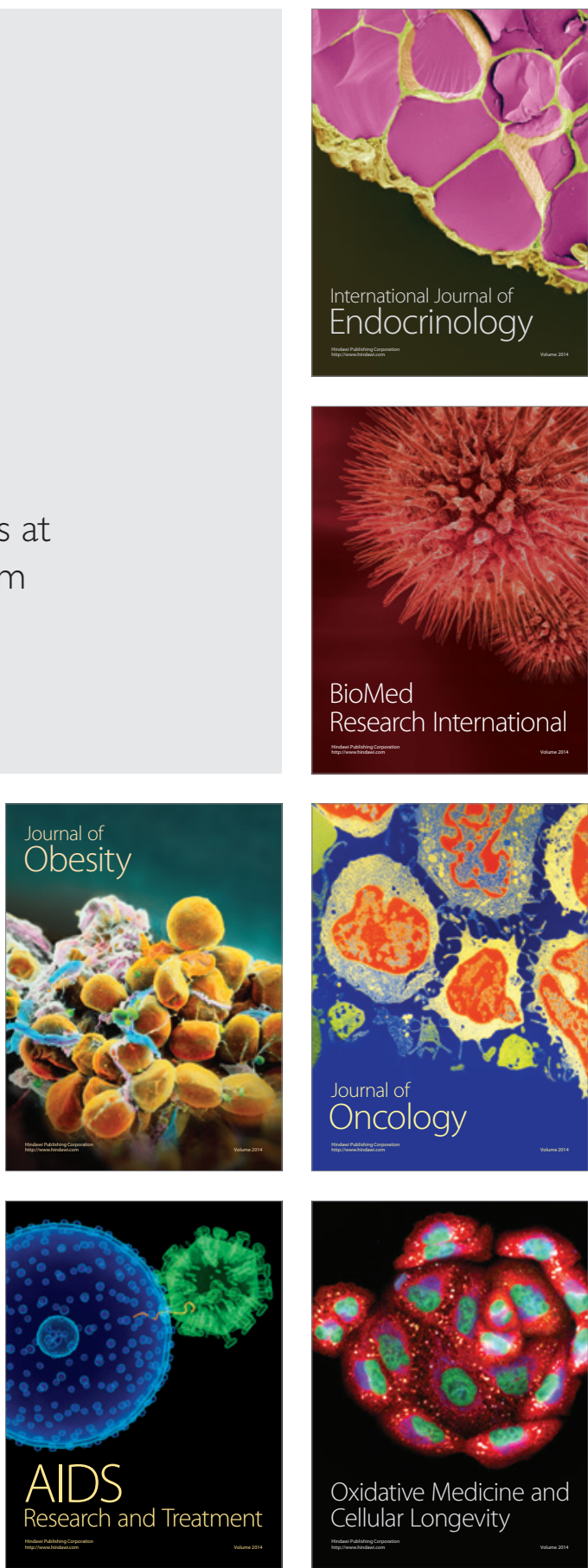